\title{
Geopolítica de los derechos humanos en las fronteras terrestres de Colombia en el primer periodo presidencial de Juan Manuel Santos ${ }^{1}$
}

\author{
Geopolitics of Human Rights on the Terrestrial Borders of Colombia \\ in the First Presidential Period of Juan Manuel Santos
}

\author{
Cristhian Fernando Sánchez Giraldo ${ }^{2}$ \\ Dulfary Calderón Sánchez ${ }^{3}$
}

Fecha de recepción: 30 de noviembre de 2016

Fecha de aprobación: 15 de marzo de 2017

\section{RESUMEN}

El subdesarrollo vigente y diferente en cada país que comparte frontera terrestre con Colombia, factor presente desde la consolidación de cada Estado-nación, ha sido, si no el mayor, uno de los factores determinantes para que el Estado no garantice la protección de los Derechos Humanos. Por un lado, los Estados son atraídos por ciertas fuerzas endógenas

1 Artículo producto del proyecto de investigación Perspectivas y prospectivas de la seguridad en Colombia, gestionado en la Facultad de Gobierno y Relaciones Internacionales, Universidad Santo Tomás. Bogotá, Colombia. DOI: http://dx.doi.org/10.15332/s1909-0528.2017.0002.02

2 Profesor titular de la Facultad de Gobierno y Relaciones Internacionales de la Universidad Santo Tomás. Bogotá, Colombia. PHD (c) Ciencia Política y Relaciones Internacionales, Universidad Complutense de Madrid, España. Magíster en Relaciones Internacionales de la Pontificia Universidad Javeriana. Bogotá, Colombia. Profesional en Relaciones Internacionales de la Universidad de Bogotá Jorge Tadeo Lozano. Bogotá, Colombia. Correo electrónico: cristhiansanchez@usantotomas.edu.co

3 Coordinadora de la Línea de Investigación en Políticas Públicas como Eje de Gobernabilidad y Gobernanza y profesora titular de la Facultad de Gobierno y Relaciones Internacionales de la Universidad Santo Tomás. Bogotá, Colombia. PHD (c) Ciencia Política y Relaciones Internacionales de la Universidad Complutense de Madrid, Espańa; Magíster en Gestión y Política Pública de la Universidad de Chile, y Politóloga, Universidad Nacional de Colombia. Bogotá, Colombia. Correo electrónico: dulfarycalderon@usantotomas.edu.co. 
influenciadas por diferentes tendencias políticas, económicas, sociales y/o culturales, las cuales se ven reflejadas en posibles conflictos sean estos estructurales o coyunturales, y, por otro lado, se encuentran diferentes fuerzas exógenas que están en constante reacción, como lo son las relaciones internacionales, determinantes también en la situación interna de cada Estado, como se demuestra en el caso de Venezuela, donde su situación interna genera inestabilidad en la geopolítica fronteriza.

A pesar de que en la mayoría de los Estados latinoamericanos hay una concordancia jurídica en su constitución política al involucrar el Derecho Internacional de los DD. HH., es evidente que su aplicabilidad está sujeta a estas fuerzas endógenas y exógenas generadoras de una fuerte deslegitimación tanto en las democracias de la región, como en sus instituciones. De ahí la urgencia de lograr un fortalecimiento democrático dentro de un marco jurídico estable, protector y garante de los DD. HH., logrando consolidar unas mejores relaciones entre los vecinos.

Palabras clave: Derechos Humanos, geopolítica, Estado, gobernabilidad, fronteras, integración, desarrollo, subdesarrollo, narcotráfico, desigualdad, pobreza, conflicto, desplazamiento, persecuciones.

\section{Abstract}

The current and different underdevelopment in each country that shares land border with Colombia, from the consolidation of each nation-state, has been if not the greatest, one of the determining factors for the state does not ensure the protection of human rights. On the one hand, States are attracted by certain endogenous forces influenced by different political, economic, social and/or cultural policy trends, which are reflected in possible conflicts are these structural or circumstantial, and, on the other hand, are different exogenous forces that are in constant reaction, such as international relations, determinants also in the internal situation in each State, as demonstrated in the case of Venezuela, where its internal situation generates instability in the geopolitical border.

Despite the fact that in the majority of Latin American States there is a legal agreement in their political constitution to involve the International Law of human rights, it is evident 
that its applicability is subject to these endogenous and exogenous forces generating a strong Rejection both in the democracies of the region, as in its institutions. Hence the urgency of strengthening democracy within a stable legal framework, protector and guarantor of human rights, managing to build better relations between the neighbors.

Keywords: Human rights, Geopolitics, State, Governance, Borders, Integration, Development, Underdevelopment, Drug trafficking, Inequality, Poverty, Conflict, Displacement, Persecution. 


\section{INTRODUCCIÓN}

Al ser concebidos como universales, los Derechos Humanos no deberían tener fronteras ni nacionalidad, puesto que están dirigidos a todos los seres humanos. Al ser universales, trascienden las fronteras y al dirigirse hacia la protección de los individuos, se deben dejar a un lado las ciudadanías y/o nacionalidades. Por lo tanto, son los Estados los que tienen la obligación de garantizar a cualquier individuo su cumplimiento, sin importar si es o no nacional del Estado en que se encuentre.

La geopolítica de los $\mathrm{DD}$. HH. supone que todos los individuos son iguales y gozan de los mismos derechos. Sin embargo, es evidente que en la práctica esta teoría universal es imperfecta y limitada, pues vivir en un país desarrollado o subdesarrollado no determina por sí mismo su garantía. Teniendo en cuenta la dinámica internacional actual, puede afirmarse que en los países desarrollados es más factible garantizar los DD. HH. a sus ciudadanos, situación contraria en los países subdesarrollados, donde existen problemas estructurales de gran importancia que impiden su cumplimiento. En el caso colombiano, la presencia de grupos al margen de la ley, el desplazamiento forzado, la pobreza y el desempleo, entre otros, dificultan su aplicabilidad.

Por lo anterior, cuando se hable de la violación a los DD. HH. no se tendrá en cuenta si es una violación contra colombianos en cualquiera de los Estados fronterizos, sino violaciones de individuos dentro de determinado Estado, demostrando el principio de extraterritorialidad que tienen.

Los DD. HH. constituyen un eje fundamental para el desarrollo humano y para la gobernabilidad de los Estados, y cuentan con un marco normativo y jurídico, así como con una serie de acciones y herramientas internacionales para su protección y garantía en los territorios.

Los desafíos e implicaciones que remite su exigibilidad en diferentes instancias dadas las dinámicas de un conflicto armado y las amenazas propias de una geografía compleja de gobernar como las fronteras terrestres de Colombia permiten que estas periferias sean susceptibles a que exista una débil presencia estatal y que a su vez, no 
permita generar una reacción inmediata frente a la vulneración y la garantía de los DD. HH. de quienes habitan en estas zonas.

Para entender las variaciones en el comportamiento de la política internacional de los países fronterizos, hay que partir del supuesto de que las percepciones y el comportamiento de la mayor parte de los Estados están influenciados por los intereses particulares y el entorno en que coexisten; de ahí que el debilitamiento latente de las democracias, así como el mantenimiento y fortalecimiento de principios tales como el de soberanía y nacionalismo, marginados desde el siglo pasado en el proceso de integración europeo, están convirtiéndose en un obstáculo para la consecución de una lucha frontal conjunta contra los problemas estructurales que afectan el desarrollo regional, donde priman las diferentes tendencias ideológicas, y en algunos casos populistas, que impiden visualizar de manera acertada la necesidad de una unión regional y continental.

En este escenario, la prolongada ausencia del Estado es aprovechada en gran medida por los grupos armados ilegales, por cuanto existe una geopolítica definida a través de sus rutas y lugares estratégicos para delinquir, exponiendo a las comunidades fronterizas a los efectos del conflicto armado, situación evidente principalmente en las fronteras con Venezuela y Ecuador, donde hay mayor concentración de población de fronteras, presentándose así una crisis humanitaria sin precedentes.

Sin dejar de lado la delimitación temporal de esta ponencia, es imposible no hacer referencia a la actual situación en la frontera entre Colombia y Venezuela, la cual ha desencadenado una evidente crisis humanitaria, política y social con la irrefutable violación de los DD. HH. de los individuos allí radicados, escenario sumamente preocupante para la estabilidad geopolítica de la región, precisamente por tratarse de la frontera más dinámica de nuestro país. 


\title{
1. LA GEOPOLÍTICA EN EL TERRITORIO DE FRONTERAS: DESAFÍOS E IMPLICACIONES
}

\author{
Existe una gran variedad de perspectivas \\ que se acogen hoy bajo la denominación de geopolitica.
}

MÉNDEZ, 2002

$\mathrm{Al}$ establecer un análisis sobre las fronteras de un Estado y los DD. HH., se tienen en cuenta los principales elementos de análisis relevantes para estudiar cualquier política exterior, como las prioridades temáticas y geográficas.

Es necesario aclarar que al analizar los $\mathrm{DD}$. $\mathrm{HH}$. desde una perspectiva de geopolítica clásica ${ }^{4}$, puede caerse en el error de no relacionarlos con lo que varios investigadores y autores plantearon en el momento de realizar sus estudios, puesto que para ellos el espacio geográfico era considerado si no el único elemento, sí el más relevante, dejando a un lado otros que para el siglo XXI se han vuelto importantes. Por otro lado, al estudiar las fronteras de los Estados no se presenta el mismo problema, puesto que la mayoría de los estudios geopolíticos consideran estas como un elemento esencial del espacio geográfico.

De ahí que para realizar un estudio relevante de la geopolítica de los DD. HH. en las fronteras terrestres de un Estado, no solo se tenga presente la definición de geopolítica cuyo elemento más importante es el territorio estatal, sino que aparte del Estado se refiera a diferentes actores, elementos, fenómenos y manifestaciones, es decir, se puede afirmar que: "la geopolítica es el estudio de las relaciones internacionales y los conflictos desde una perspectiva geográfica” (Braden \& Shelley, 2000).

4 Para Kristof (1960), la “geopolítica” será lo que significa la etimología de la palabra: la política geográfica, es decir, la política interpretada geográficamente. Cuarenta años atrás Kjellen (1916), había definido la geopolítica en términos de ciencia del Estado como organismo geográfico, tal como se refleja en el espacio. 
Como ya se mencionó, al ser concebidos como universales, los Derechos Humanos no deberían tener fronteras ni nacionalidad, puesto que están dirigidos a todos los seres humanos.

Son valores universales y garantías jurídicas que protegen a personas y grupos contra acciones y omisiones primordialmente de agentes del Estado que interfieren con las libertades fundamentales, los derechos y la dignidad humana. El espectro completo de los Derechos Humanos implica el respeto, la protección y el cumplimiento de los derechos civiles, culturales, económicos, políticos y sociales, así como el derecho al desarrollo[...] son universales, es decir, pertenecen intrínsecamente a todos los seres humanos, y son interdependientes (ONU, 2017).

$\mathrm{Al}$ ser universales, los DD. HH. trascienden las fronterasy al dirigirse hacia la protección de los individuos, se deben dejar a un lado las ciudadanías y/o nacionalidades. Por lo tanto, son los Estados los que tienen la obligación de garantizar a cualquier individuo su cumplimiento, sin importar si es o no nacional del Estado en que se encuentre, de modo que si un individuo no es ciudadano de un Estado, esto no implica su desprotección.

Aunque el análisis de los DD. HH. desde una perspectiva geopolítica, en la cual todos los individuos son iguales y gozan de los mismos derechos, puede entenderse como idealista, es necesario hacer hincapié en lo sesgada que se encuentra la praxis de esta teoría universal de los DD. HH., puesto que cada vez que se estudia, parece referida a una sociedad de ficción, donde la paz jamás sería expuesta a la amenaza de una guerra o conflicto. Sin embargo, más peligroso que este idealismo es la indiferencia con que los Estados dejan a sus propios ciudadanos al no garantizar el derecho internacional de los DD. HH., pues es evidente que en la praxis esta teoría es imperfecta y relativa, toda vez que vivir en un país desarrollado o subdesarrollado no determina por sí mismo la garantía de dichos derechos.

5 Véase, por ejemplo, la Carta de las Naciones Unidas, Art. 55 c), la Declaración Universal de Derechos Humanos, art. 2, y la Declaración y Plan de Acción de Viena. 
No obstante, es más factible la garantía de los DD. HH. en los países desarrollados, a diferencia de los países subdesarrollados, puesto que en estos últimos coexisten problemas estructurales de gran importancia en donde pierden su relevancia. Como ya se mencionó, es el caso colombiano donde fenómenos como la presencia de grupos al margen de la ley, el desplazamiento forzado, la pobreza y el desempleo, entre otros factores, impiden que dichos DD. HH. se garanticen, agudizando su vulnerabilidad. Por lo anterior, cuando se hable de la violación a los DD. HH. no debe pensarse si es una violación contra colombianos en cualquiera de los Estados fronterizos, sino violaciones de individuos dentro de determinado Estado, demostrándose así el principio de extraterritorialidad que poseen.

Al mismo tiempo, debe entenderse que la violación de los DD. HH. dentro de cualquier territorio afecta en gran medida la perspectiva geopolítica tanto en el Estado en que se comete dicha violación, como en cualquier Estado fronterizo, dado a las relaciones que se tejen a nivel histórico y cultural que permiten evidenciar elementos estructurales y coyunturales dentro de los marcos de acuerdos, tratados o pactos existentes entre estos. Por lo anterior, el efecto que puede tener la violación de los derechos dentro de un territorio afectará en mayor medida a sus vecinos que generalmente son quienes han mantenido esta relación histórica. Siendo los principales receptores indirectos de las consecuencias de esta trasgresión.

La responsabilidad que tienen los Estados en este sentido es absoluta, tomando como referencia la ya mencionada Teoría Universal de los DD. HH., puesto que estos deben proteger a cualquier persona, sin importar su origen. Obviamente para los Estados es una labor imposible de cumplir, así exista un compromiso formal, firmado ante organismos internacionales y ratificado por los parlamentos. En el caso colombiano, estos problemas se ven reflejados frente a los países fronterizos por los siguientes factores:

\subsection{Ausencia del Estado}

Que es aprovechada en gran medida por los grupos armados ilegales, por cuanto existe una geopolítica definida a través de sus rutas y lugares estratégicos para delinquir, exponiendo a las comunidades fronterizas a los efectos del conflicto armado, 
situación evidente principalmente en las fronteras con Venezuela y Ecuador, donde hay mayor concentración de población de fronteras, presentándose así una crisis humanitaria que, en materia de seguridad, trajo consecuencias nefastas, cuando los Nuevos Grupos Armados Ilegales (NGAI) y sucesores de los paramilitares ${ }^{6}$ y los grupos guerrilleros trataron de reubicarse geoestratégicamente,

La salida de colombianos hacia el exterior se registra por lo menos desde la década de los ochenta, pero la aceleración del conflicto provoca un fuerte crecimiento en el número de personas que buscan protección internacional [...] La militarización de las fronteras no ha logrado avances sostenibles en materia de seguridad, y los esfuerzos de la fuerza pública para aumentar la confianza de la población local continúan siendo socavados por los abusos contra los Derechos Humanos y las violaciones del DIH (Informe de Crisis Group Sobre América Latina, 2011, p.29)”.

\subsection{Economías ilícitas}

Las regiones fronterizas se fueron convirtiendo en zonas favorables para las economías ilícitas quedando expuestas a las acciones de los grupos armados ilegales. En cada célula de cualquier grupo narcotraficante existe también una geopolítica definida a través de sus rutas y lugares estratégicos para la producción, distribución y consumo, por lo que en el proceso de producción y tráfico de estupefacientes, participan gran variedad de actores y elementos que hacen que para el Estado sea muy difícil el control de estas zonas. Las zonas fronterizas con Panamá, Venezuela y Ecuador son importantes puntos donde ingresan armas ilegales, las cuales en la mayoría de los casos se intercambian por drogas (Codhes, 2005; Cragin \& Hoffman, 2003).

6 Nuevos Grupos Armados Ilegales y sucesores del paramilitarismo, término genérico para describir a los grupos armados ilegales que han emergido tras el fin de la desmovilización de los paramilitares en 2006. 


\subsection{Corrupción}

La inminente corrupción, que se encuentra presente en todos los estamentos del Estado, ha sido un fenómeno que ha quebrantado los esfuerzos por construir instituciones locales fuertes.

\subsection{Pobreza estructural}

Las zonas de fronteras se han convertido paulatinamente en escenarios de pobreza estructural y coyuntural donde coinciden personas que tuvieron que abandonar en la mayoría de los casos sus lugares de origen y asentarse (legal o ilegalmente) en estos sitios para huir de la violencia o simplemente para buscar mejorar su calidad de vida.

\subsection{Desplazamiento}

Desplazamiento interno de individuos que se da hacia diferentes regiones colombianas, cobra su extraterritorialidad cuando se traspasan las fronteras, convirtiéndose en un desplazamiento externo, dado por la violencia, falta de oportunidades, pobreza, desigualdad, conflicto interno, persecuciones políticas, sociales y cultuales.

\subsection{Complejidad}

La región fronteriza es un escenario donde convergen una serie de actividades cotidianas, convirtiéndose en puntos de encuentro, económicos, sociales, y culturales, y a través de las relaciones sociales se establecen vínculos de pertenencia hacia una zona común, puesto que para sus habitantes esos límites fronterizos no deberían existir. Por esta misma cotidianidad, los Estados fronterizos deben garantizar ese esquema de convivencia, sin desproteger los DD. HH. Por ello, los Estados deben fortalecer y proteger estas relaciones de vecindad,

Aún hacen falta espacios e instituciones eficaces para la solución mancomunada de problemas y la promoción del desarrollo fronterizo. Esto refleja en parte la reticencia de los vecinos a reconocer alguna responsabilidad en un conflicto que, a su juicio, es un asunto interno de Colombia, pero que en realidad se 
sostiene gracias a redes criminales transnacionales y que cobra cada vez más víctimas en cualquier lado de las fronteras. (Informe Sobre América Latina, 2011, p. 2)

\subsection{Intereses particulares}

Las percepciones y el comportamiento de la mayor parte de los Estados están influenciados por los intereses particulares y el entorno en el que coexisten; de ahí que el debilitamiento latente de las democracias, así como el mantenimiento y fortalecimiento de principios tales como el de soberanía y nacionalismo están convirtiéndose en un obstáculo para una lucha frontal conjunta contra los problemas estructurales que afectan el desarrollo regional, donde priman las diferentes tendencias ideológicas, y en algunos casos populistas, que impiden visualizar de manera acertada, la necesidad de una unión regional y continental.

\subsection{Ideologías}

Existe un armazón político que, sin lugar a dudas, no permite que la integración fronteriza se consolide, situación absurda y retrógrada que ha demostrado que a pesar de que son menos las diferencias que nos unen entre los vecinos, no hemos podido siquiera superar los siguientes escalones para alcanzar la tan anhelada integración total.

\subsection{Falta de liderazgo}

La necesidad que tienen los países que comparten fronteras con Colombia de tratar de alcanzar conjuntamente un liderazgo global y la estrategia que trae consigo el actuar en bloque, para así conseguir que cada Estado logre fortalecer tanto sus canales multilaterales, como los bilaterales dentro de su política exterior. 


\section{Los Derechos Humanos como eJE DE Gobernabilidad EN LAS FRONTERAS}

En preámbulo de la Declaración Universal de Derechos Humanos se estipula:

Como ideal común por el que todos los pueblos y naciones deben esforzarse, a fin de que tanto los individuos como las instituciones, inspirándose constantemente en ella, promuevan, mediante la enseñanza y la educación, el respeto a estos derechos y libertades, y aseguren, por medidas progresivas de carácter nacional e internacional, su reconocimiento y aplicación universales y efectivos, tanto entre los pueblos de los Estados miembros como entre los de los territorios (ONU, 1948).

En esta medida, los DD. HH. deben trascender las fronteras nacionales toda vez que sus características de ser universales, indivisibles, obligatorios, no negociables e irreversibles y progresivos los hacen interdependientes, complementarios y no jerarquizados por lo que al ser violado un derecho se desencadena una serie de derechos vulnerados.

Colombia tiene una característica especial y es que de sus 32 departamentos, once tienen fronteras terrestres con sus países vecinos: Ecuador, Panamá, Venezuela, Brasil y Perú. Cabe señalar que los habitantes de estas fronteras no solo comparten el territorio, sino que crean construcciones sociales, económicas, políticas y culturales que genera vínculos más allá de los conceptos nacionalistas que pueden suscitarse.

De la población que abarca el territorio fronterizo, el 94,5\% se concentra en la frontera con Venezuela y Ecuador, lo que implica que exista mayor atención a las políticas sociales y políticas públicas para subsanar las necesidades propias del territorio. Así mismo, se deben precisar y fortalecer los programas con enfoque diferencial dado a que los grupos etarios y los grupos étnicos que habitan allí, son más vulnerables a la trasgresión de sus derechos, como consecuencia por la ausencia del Estado. 

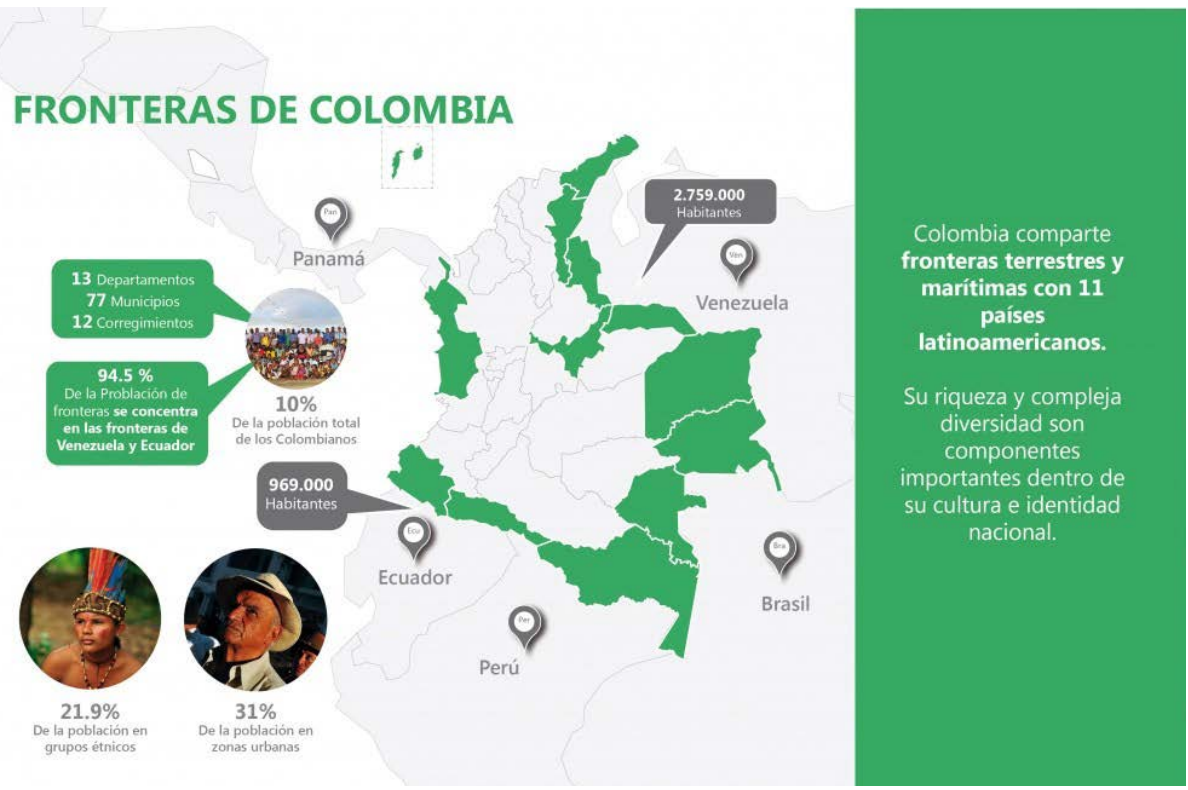

Fuente: Ministerio de Relaciones Exteriores (2013). Documento Plan de Fronteras para la prosperidad, agosto de 2013. Tomado de http://www.cancilleria.gov.co/prosperity

$\mathrm{Al}$ asumir como Jefe del Estado, en su primer periodo presidencial, en ceremonia realizada en la Plaza de Bolívar de Bogotá, el 7 de agosto de 2010, Juan Manuel Santos manifestó que "se debía construir una relación de respeto y cooperación con los vecinos y reducir el abismo de desarrollo entre la periferia y el centro del país" (Santos, J.M., 2010). Al analizar no solo las anteriores palabras, sino en general el discurso completo, puede evidenciarse que dada la coyuntura en que se encontraban las relaciones diplomáticas con los países vecinos, en especial con Ecuador luego de los bombardeos al campamento de Raúl Reyes en su territorio, debían restaurarse estas relaciones. Con Venezuela se estipuló una agenda igualmente prioritaria en materia de relaciones exteriores, de modo que las fronteras terrestres eran tema primordial para el Gobierno. Pese a estos esfuerzos por restablecer las relaciones diplomáticas con los vecinos, los incidentes en las fronteras terrestres persisten: 
En la medida en que la intensidad del conflicto armado no disminuye, la situación humanitaria es grave a lo largo de la frontera sur. En ambos lados, el incremento en la presencia militar fue, en parte, una estrategia para mantener y profundizar su control territorial, pero los avances en materia de seguridad o nunca se materializaron para la mayoría de los ciudadanos, en el caso de Narińo, o se han estancado, pues el nivel de violencia sigue alto a ambos lados de la frontera (Informe de Crisis Group sobre América Latina, 2011).

El plan de Desarrollo 2010-2014, "Prosperidad para todos", esboza un marco político que se encuentra orientado específicamente a que exista un cierre de la brecha entre el centro y la periferia de Colombia, tomando como eje el desarrollo y la seguridad de los habitantes de las fronteras terrestres del Estado. Para ello se genera el Plan de Fronteras para la Prosperidad en 2013 que tiene como principios: el desarrollo sustentable, el desarrollo humano, una política pública diferenciada, una coordinación y liderazgo central con visión local, un enfoque local y sostenible y la amplia participación (Cancillería, 2013).

Sin embargo, la dinámica del conflicto, la precaria presencia del Estado, el contrabando, las mafias y los actores armados, entre otros problemas, hacen que este territorio fronterizo genere capítulos de violencia que han afectado a sus habitantes y vulnerado los DD. HH., motivando en ocasiones un desplazamiento y una migración a los países fronterizos como mecanismo de protección a sus derechos fundamentales y a los de su núcleo familiar. Para Socorro Ramírez (2015):

En la mayor parte de los intentos se observan avances en la concepción de fronteras si se la compara con la predominante en algunos países vecinos donde, por conflictos territoriales y porque los militares derivan su poder del papel en las fronteras, las definiciones al respecto se centran en consolidar el límite como barrera y perímetro de seguridad del Estado.

Seguridad que el Estado debe brindar para los habitantes del territorio nacional y sus vecinos fronterizos, pero que en el caso de Ecuador, con la frontera colombiana la comunidad y líderes denuncian: 
Violaciones a los derechos de las personas como la restricción a la movilidad humana, el señalamiento de ser encubridores o cómplices de la guerrilla de las FARC, la búsqueda de personas en territorio ecuatoriano o detenciones arbitrarias e irregulares; mientras que en territorio colombiano el Ejército y fuerza naval colombianos persisten en la persecución y señalamiento a líderes y defensores comunitarios de Derechos Humanos (Asamblea comunitaria binacional de organizaciones y comunidades campesinas e indígenas de la provincia de Sucumbíos y Puerto Asís, 2014).

En el mismo comunicado, la Asamblea comunitaria binacional menciona que existe una intensificación del conflicto colombiano por las acciones extractivas de compañías petroleras, toda vez que con estas viene la instalación de bases militares para su seguridad, lo cual genera mayores confrontaciones entre los ejércitos irregulares y las tropas colombianas quedando la población civil en la mitad de los enfrentamientos y hostigamientos.

En este contexto, no es ajeno el caso de las fronteras con Venezuela y Ecuador dada a las características particulares que tiene el conflicto armado colombiano y al debilitamiento en la presencia de ambos Estados que ha generado la continuidad de los corredores para la distribución de drogas y la ilegalidad que han constituido mafias amenazando la estabilidad social y económica entre los habitantes de la frontera.

Cuando se hace referencia a las acciones del Estado frente a las demandas sociales es necesario remitirse a la gobernabilidad que en términos de Antonio Camou (2001), debe ser entendida como:

Un estado de equilibrio dinámico entre el nivel de las demandas societales (sic) y la capacidad del sistema político (Estado/gobierno) para responderlas de manera legítima y eficaz. Ello permite superar una lectura dicotómica (gobernabilidad versus ingobernabilidad) y analizar grados y niveles de gobernabilidad involucrando en la definición una "serie de 'acuerdos' básicos entre las élites dirigentes [...] en torno a tres ámbitos principales [...] el nivel de la cultura política [...] el nivel de las reglas e instituciones del juego político [...] (y) acuerdos en torno al papel del Estado y sus políticas públicas estratégicas. 
Cuando se hace referencia a una violación sistemática de DD. HH., producto de situaciones propias del territorio, la gobernabilidad del Estado debe emprender acciones afirmativas a partir de políticas sociales, políticas estatales, políticas públicas y cooperación fronteriza que permitan generar planes y proyectos de mediano y largo plazo hacia la integración social generando una ratificación en la presencia del Estado.

En el informe al Congreso de la República en 2014, el presidente Juan Manuel Santos señala que "Con el propósito de atender los problemas generados por el contrabando, se incrementará la vigilancia y el control policial y aduanero de las fronteras, mediante la dotación de medios de observación, comunicaciones, protección y judicialización. También se mejorará la infraestructura y los medios de control, contención y vigilancia en los puntos de paso de personas y mercancías".

En el mismo informe se mencionan acciones de control migratorias, programas para las denominadas subregiones y una inversión de $\$ 3,4$ billones hacia el 2021 en proyectos de desarrollo agropecuario, mineral, fluvial y ambiental. Con lo cual se espera mayor presencia del Estado y un desarrollo óptimo para los habitantes de las fronteras terrestres.

\section{Conclusiones}

Es evidente la necesidad que tienen los países que comparten fronteras con Colombia de alcanzar un liderazgo global y actuar en bloque en aras de que cada Estado logre el fortalecimiento de sus canales multilaterales dentro de su política exterior. Sin embargo, es indiscutible que ha existido un armazón político que impide que dicha unión se presente, lo cual constituye una situación absurda y retrógrada que evidencia que a pesar de que son menos las diferencias que las similitudes que nos unen, no hemos podido siquiera superar los siguientes escalones para alcanzar la tan anhelada integración total.

Para entender las variaciones en el comportamiento de la política internacional de los países fronterizos hay que partir del supuesto de que las percepciones y el comportamiento de la mayor parte de los Estados están influenciados por los intereses 
particulares y el entorno en el que coexisten; de ahí que el debilitamiento latente de las democracias, así como el mantenimiento y fortalecimiento de principios tales como el de soberanía y nacionalismo, marginados desde el siglo pasado en el proceso de integración europeo, están convirtiéndose en un obstáculo para la consecución de una lucha frontal conjunta contra los problemas estructurales que afectan el desarrollo regional, donde priman las diferentes tendencias ideológicas, y en algunos casos populistas, que impiden visualizar de manera acertada la necesidad de una unión regional y continental.

El problema de los DD. HH. en las fronteras terrestres colombianas debe atenderse con unas instituciones capaces de mitigar y subsanar las necesidades de la población civil que se encuentra no solo en la dinámica estatal, sino en instancias exógenas, como los problemas propios de los países fronterizos que se generan a partir de la ausencia de los Estados y de la misma geopolítica territorial. Ello implica el conocimiento de las dinámicas de integración regional y de las oportunidades que puede tener el continente americano:

Es muy importante contribuir a la creación de un tejido social fronterizo que confirme la clara intención de Colombia de mantener una actitud sostenida de "buena vecindad" y que reconozca que, en la mayoría de los casos, opera mejor en frontera la relación directa entre los alcaldes, los gobernadores, las instituciones académicas y las autoridades de policía, para el desarrollo de iniciativas conjuntas [...] Las zonas de frontera deben concebirse como polos de desarrollo para consolidar la buena vecindad con los países limítrofes, en beneficio del interés nacional y el mejoramiento de las condiciones de vida de las comunidades asentadas en la región. En consecuencia, la integración y el desarrollo fronterizo deben preservarse como un principio central de la política exterior colombiana, a partir del cual se reconozca su importancia geoestratégica. En tal sentido, cualquier iniciativa para las fronteras debe ser consecuente con las necesidades coyunturales y estrictamente locales de estas regiones, y con los desafíos compartidos que surgen de la convivencia propia en las líneas imaginarias que separan a los Estados (Soto, 2010). 


\section{REFERENCIAS}

Aldonas, Grant (2008). The future of Regional Integration in the Americas. Paper preparado para el BID sobre prioridades de la integración regional.

Asamblea comunitaria binacional de organizaciones y comunidades campesinas e indígenas de la provincia de Sucumbíos (Ecuador) y Puerto Asís (Colombia) (2014). Situación de Derechos Humanos en la frontera colombo ecuatoriana en: http://utopialapalabra. blogspot.com.co/2014/05/violan-derechos-humanos-de-comunidades.html

Braden, K.E. \& Shelley, F.M. (2000). Engaging geopolitics. Londres: Prentice Hall.

Camou, A. (2001). Los desafíos de la gobernabilidad. Estudio preliminar y compilación. México: Flacso/IISUNAM/Plaza y Valdés.

Codhes, (2005). Situación de conflicto y desplazamiento en las fronteras: el cerco se cierra. Bogotá.

Comisión Económica para América Latina y el Caribe, CEPAL (2009). Panorama económico y social de ALC (2008-2009).

Cragin, Kim \& Hoffman, Bruce (2003). Arms Trafficking and Colombia. Santa Mónica.

Fescol, (2008). "La búsqueda de nuevos escenarios de integración para Colombia”. En: Policy paper. N.o 33.

Group, Crisis (2011). "Más allá de las ganancias fáciles: las fronteras de Colombia”. Informe de Crisis Group sobre América Latina. N. ${ }^{\circ}$ 40, 31 de octubre de 2011.

Kristof, L.D. (1960). "The origins and evolution of geopolitics”. The Journal of Conflict Resolution. Vol. 4, n.o 1, The Geography of Conflict (Mar., 1960), pp. 15-51.

Lee, Nancy (2008). Now More than Ever: The Case for a New Integration Strategy for the Americas. Paper preparado para el BID sobre prioridades de la integración regional. BID. 
Magariños, Gustavo (2007). Estudio sobre el espacio de libre comercio en la ALADI. Secretaría General de la ALADI en el marco del Proyecto de Cooperación técnica Regional ALADIBID. Recomendaciones técnicas para la creación del Espacio de Libre Comercio.

Méndez, Ricardo. (2010). El nuevo mapa geopolítico del mundo. Valencia: Editorial Tirant lo Blanch.

Oficina del Alto Comisionado de las Naciones Unidas para los Derechos Humanos, ACNUDH, (2008). "Los Derechos Humanos, el terrorismo y la lucha contra el terrorismo". Folleto Informativo No. 32. Genève 10, Suiza.

Organización de las Naciones Unidas, ONU (2017). Carta de las Naciones Unidas. En http:// www.un.org/es/sections/un-charter/chapter-ix/index.html

Pineda, Saúl (2009). Propuestas para fortalecer la integración regional en el actual contexto hemisférico y global. Documento preparado para la Red Latn. Lima, Perú.

PNUMA (2007). "Perspectivas del medio ambiente mundial". Geo 4. Medio ambiente para el desarrollo.

Ramírez, S. (2015). Politica de fronteras: avances y pendientes. En http://www.razonpublica.com/index.php/internacional-temas-32/7834-pol\%C3\%ADtica-de-fronterasavances-y-pendientes.html

República de Colombia. Ministerio de Relaciones Exteriores de Colombia (2007-2009). Documentos informales de trabajo.

República de Colombia. Presidencia de la República (2014). Informe al Congreso. Santos, Juan Manuel. (2014).

República de Colombia. Presidencia de la República (2014). ¡Le llegó la hora a Colombia! Discurso del presidente Juan Manuel Santos Calderón, al asumir como Jefe del Estado en ceremonia realizada en la Plaza de Bolívar de Bogotá el 7 de agosto de 2010. 
Secretaría General de la Comunidad Andina (2006). Visión Andina de la Comunidad Sudamericana. Documento interno de trabajo.

Soto, Guillermo \& otros (2010). Propuestas de Política Pública Colombia 2010/2014. Colombia: Editorial CAF, Fedesarrollo.

Soto, Guillermo \& otros (2004). La Ilusión posible. Un testimonio sobre la politica exterior colombiana. Bogotá: Editorial Norma. 